\title{
Automatic Train Control System Using Fuzzy Logic Controller
}

\author{
Meghna Madhava, N. Meghana, Mulpuru Supriya, Divya and \\ Siddalingesh S. Navalgund
}

\begin{abstract}
In this paper an attempt has been made to explain automatic train operation using fuzzy logic controller. The breaking system and Torque detection based on fuzzy logic controller. Speed, distance, weight and direction of the train are indicated and resultant graphs are shown. This paper mainly focuses on a new approach to braking system by making use of artificial intelligence applied to it. The intelligence of braking is provided by a fuzzy logic controller. The fuzzy logic controller is simulated using MATLAB. Thus the train stops at station smoothly and automatically.
\end{abstract}

Index Terms--- Braking System, Torque, Fuzzy Logic Controller, Train Operation.

\section{INTRODUCTION}

A good transport system is the index of every country. It is essential requirement of every country to travel and transport their goods fastest, safest and economical way. [1] gives the idea that in a very big country like India transportation plays a very important role because the distance between various place is more. Therefore economical and safest transportation is required. Day to Day new advancements in railways paves way for a new approach to reduce the manpower and to effectively automate the system using advanced control equipments. Generally the design of automatic braking system becomes more complex but it can be made easier and flexible by using fuzzy logic controller. [2] explains that, In order to get the outputs, necessary inputs are chosen for the fuzzy controller. The numerical inputs are converted into fuzzy sets with the help of fuzzification technique. The output fuzzy value of the fuzzy sets is converted into numerical values by the de fuzzification technique. This numerical value which is the output of the fuzzy logic controller is used to control the Torque and the braking system of the train.

\section{LITERATURE SURVEY}

The primary reason for carrying this paper based on fuzzy logic controller is that, it explains the working of a controller

Meghna Madhava, $4^{\text {th }}$ Year, Student, Dept. of E\&CE, SDMCET, Dharwad.

N. Meghana, $4^{\text {th }}$ Year, Student, Dept. of E\&CE, SDMCET, Dharwad.

Mulpuru Supriya, $4^{\text {th }}$ Year, Student, Dept. of E\&CE, SDMCET, Dharwad. Divya, $4^{\text {th }}$ Year, Student, Dept. of E\&CE, SDMCET, Dharwad.

Siddalingesh S. Navalgund, Faculty, Dept. of E\&CE, S. D. M. College of Engineering and Technology, Dharwad, Karnataka.

DOI:10.9756/BIJRCE.8201 in an understandable language.[3] explains the idea of fuzzy logic and how it can be implemented in Engineering applications. The two key features of fuzzy logic controller include: (a) A mathematical formalism for representing human knowledge involving vague concepts, and (b) a natural but effective mechanism for systematically formulating costeffective solutions to complex problems characterized by uncertainty or imprecise information. In paper [4] the general idea of automatic train operation based on MATLAB. This paper explains Automatic Train Stop(ATS) and Automatic Warning System(AWS). It is based on the Data Oriented Control Method (DOME) to achieve train control. [5] gives the idea of automatic braking system in trains by using artificial intelligence technique. The intelligence is provided by a fuzzy logic controller. The results of this paper shows that the braking system output is smooth and the train comes to stop when the distance reduces to zero. The proposed fuzzy logic helps to reduce the man power for train operation.

\section{Method Proposed}

A method to control the operation of the train with respect to speed, weight, distance, direction, torque and braking system.

For a given set of inputs membership functions has been allotted to get the desired outputs. Speed has been given membership functions as very low, low, medium, high, very high. Weight has been given membership functions as very low, low, medium, high, very high. Distance as very close, close, and far, very far and direction as forward, backward and stop.

The outputs were also assigned with the membership functions with torque as very low, low, medium, high, very high and braking system as very light, light, heavy and very heavy.

A set of rules were written which will satisfy all the possible conditions and give the required outputs.

The rules are written as if (speed is very low) and (distance is very close) then braking system is light.

The input numerical values are converted to fuzzy set by fuzzification and again sets are converted to numerical by de fuzzification. 


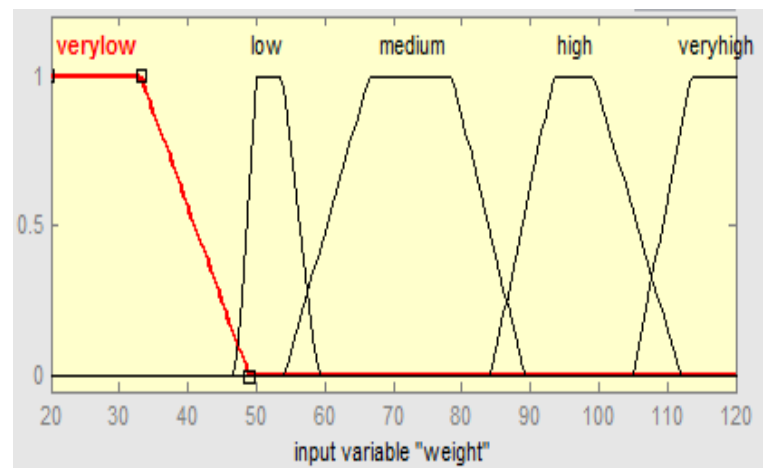

Figure 1: Membership Functions of Variable Weight

Fig.1 shows membership functions of variable weight with very low, low, medium, high, very high as various membership functions ranging till 1000 tones.

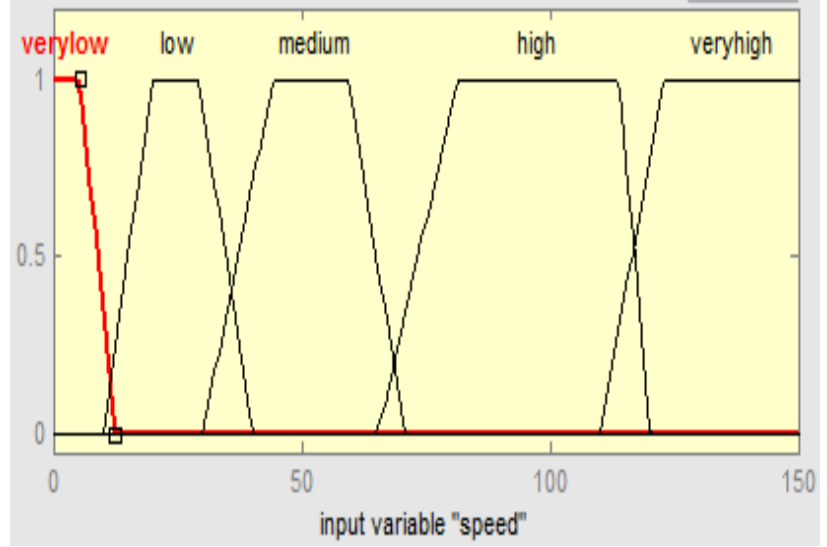

Figure 2: Membership Functions of Variable Speed

Fig. 2 shows membership functions of variable speed with very low, low, medium, high, very high as various membership functions ranging $150 \mathrm{Km}$.

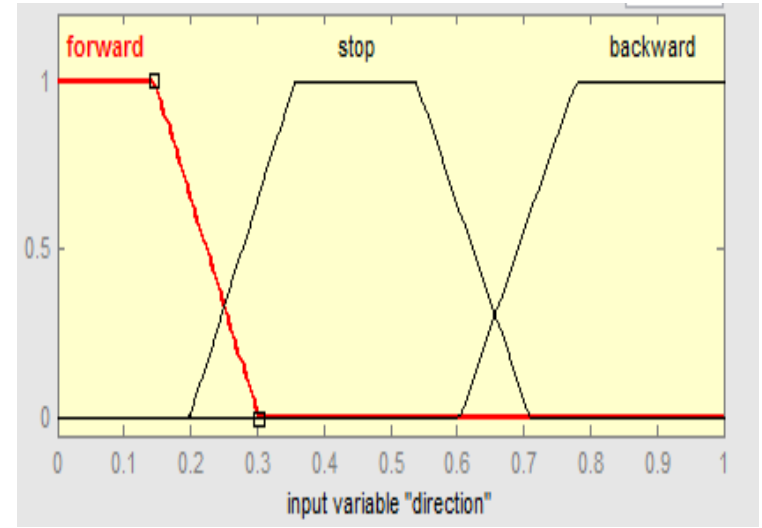

Figure 3: Membership Functions of Variable Direction

Fig. 3 shows membership functions of variable direction with forward, stop, backward as membership functions.

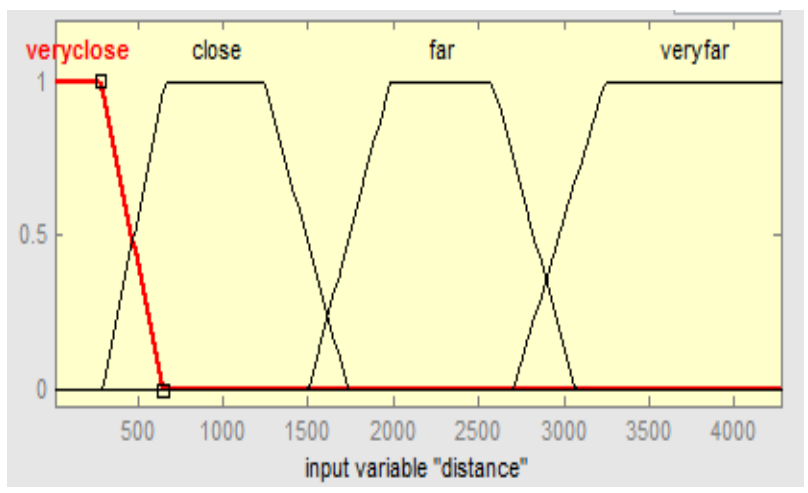

Figure 4: Membership Functions of Variable Distance

Fig. 4 shows membership functions of variable weight with very close, close, far, very far as various membership functions ranging till $1000 \mathrm{Km}$.

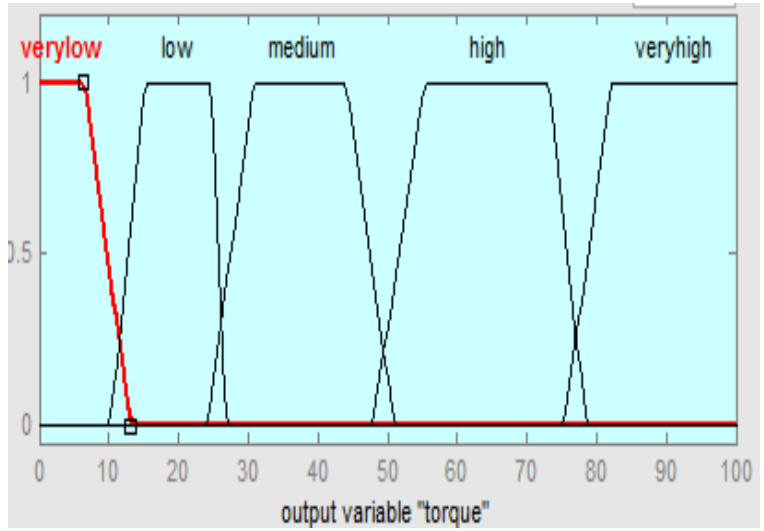

Figure 5: Membership Functions of Variable Torque

Fig. 5 shows membership functions of output variable torque with very low, low, medium, high, very high as various membership functions ranging till $100 \mathrm{~N}$.

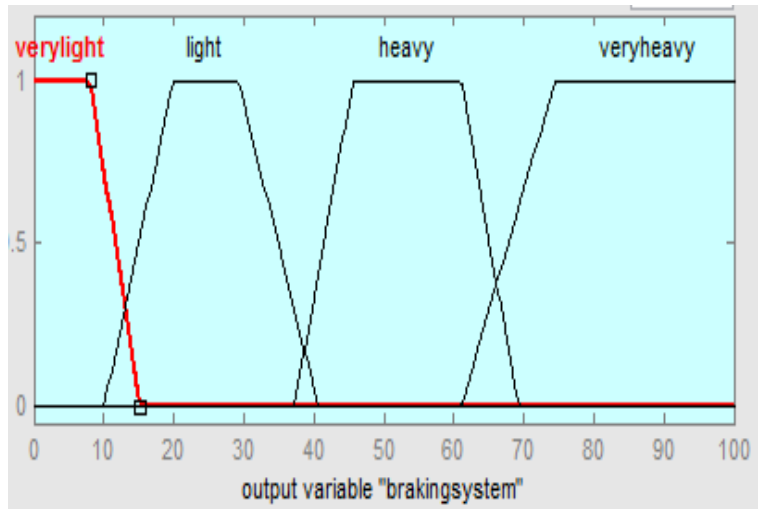

Figure 6: Membership Functions of Variable Braking System(\%)

Fig. 6 shows membership functions of output variable braking system with very light, light, heavy, very heavy as various membership functions ranging till 100 percent.

All the figures are in trapezoidal form with some probabilities occurring between the member functions. 
File Edit View Options

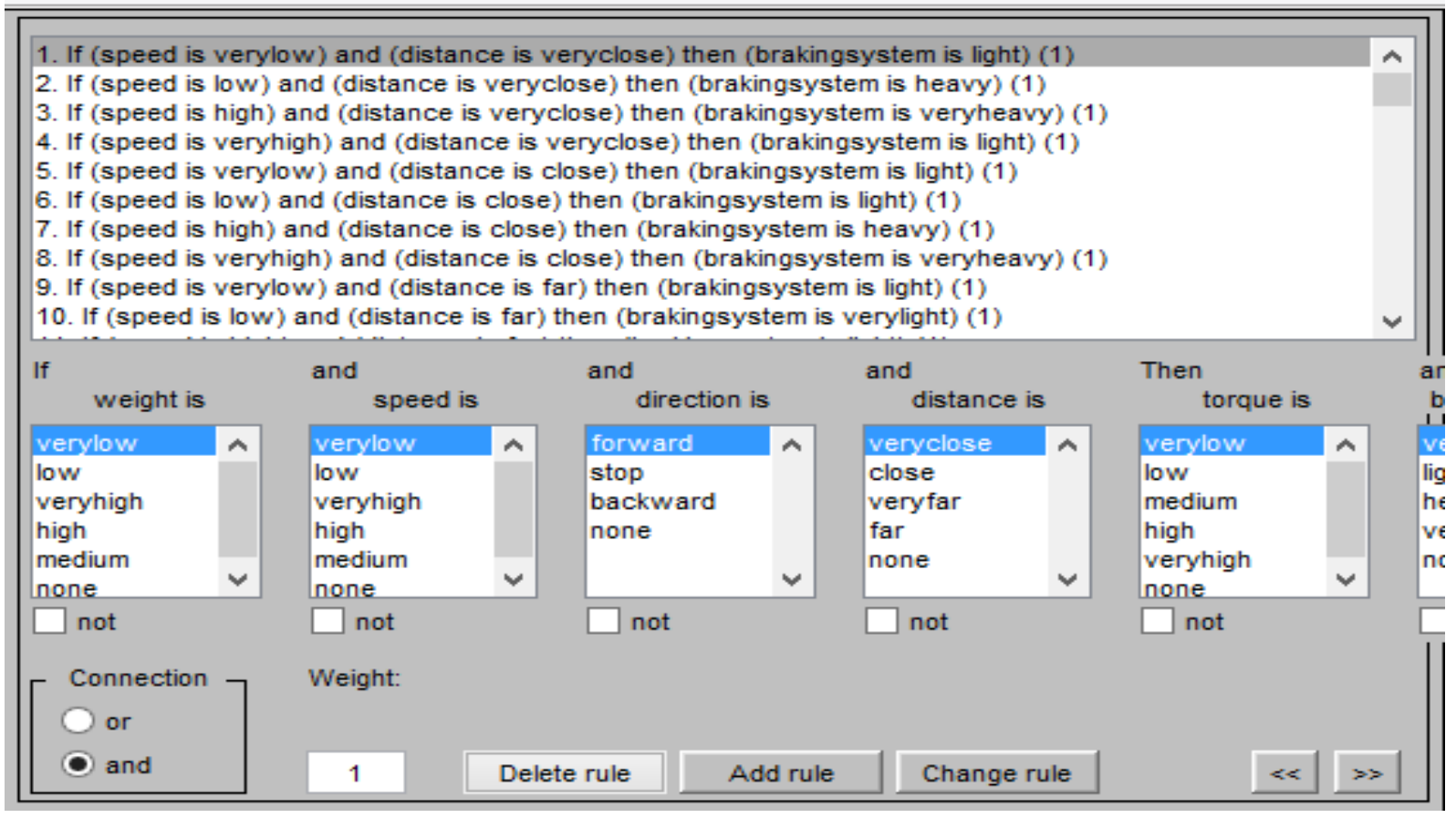

Figure 7: Rule Base

fig 7 Shows the Rules with Respect to Outputs and inputs. Several Combination of Rules are Written.

VII. RESULT AND DISCUSSION

For the implementation of the results, MATLAB R2011b (7.13.0.564) version has been used. It is a 64-bit software with License Number: 161052. Within this software there are two versions under fuzzy logic controller. They are: (a) Mamdani (b) Sugeno.

For carrying this paper Mamdani version has been used.

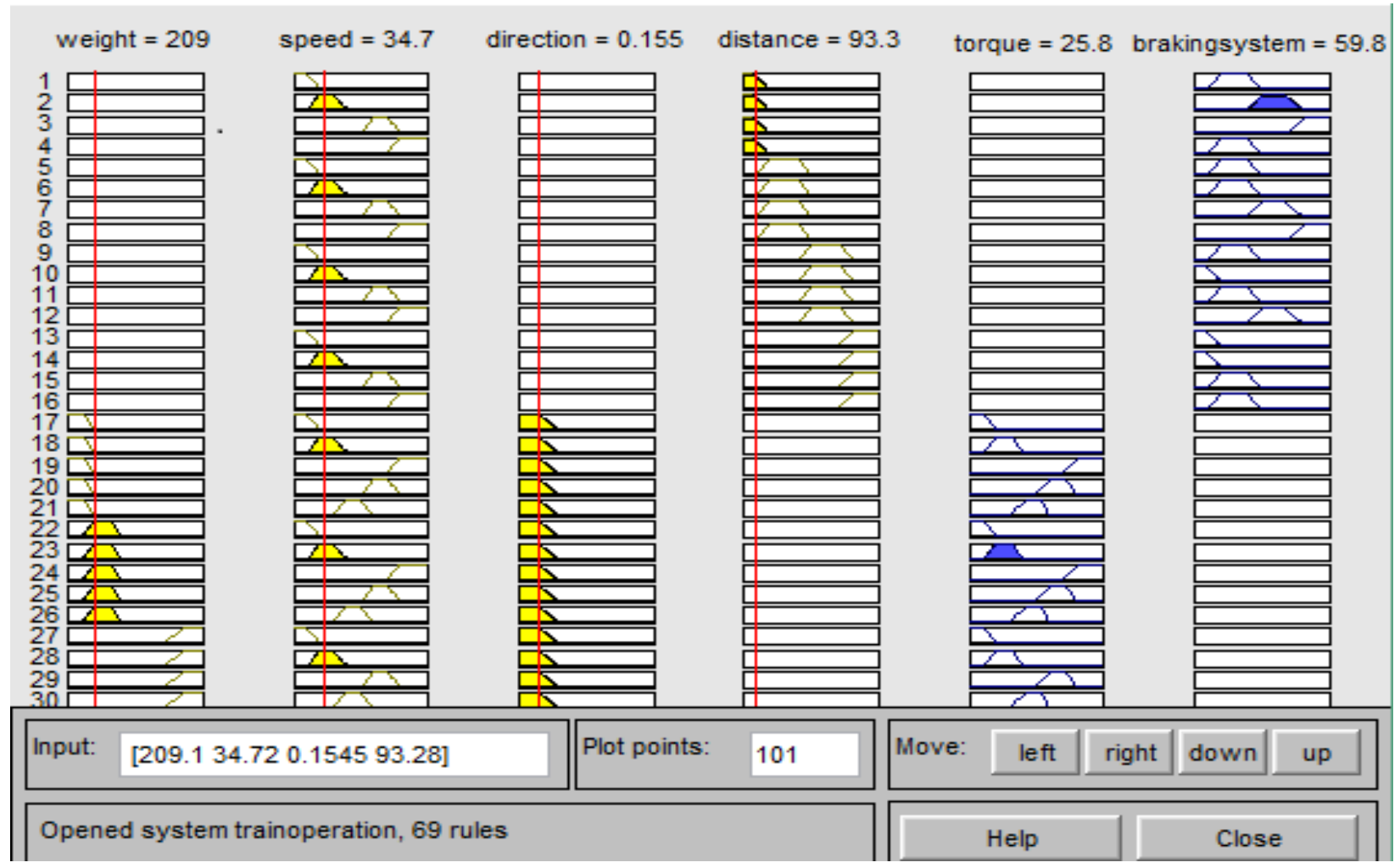

Figure 8: Rule Viewer Corresponding to Inputs and Outputs 
Fig 8 shows rules regarding the inputs and outputs. In this case the weight along with speed is kept low, direction as forward and distance as very close, so the resultant torque appears to be low and braking system as heavy.

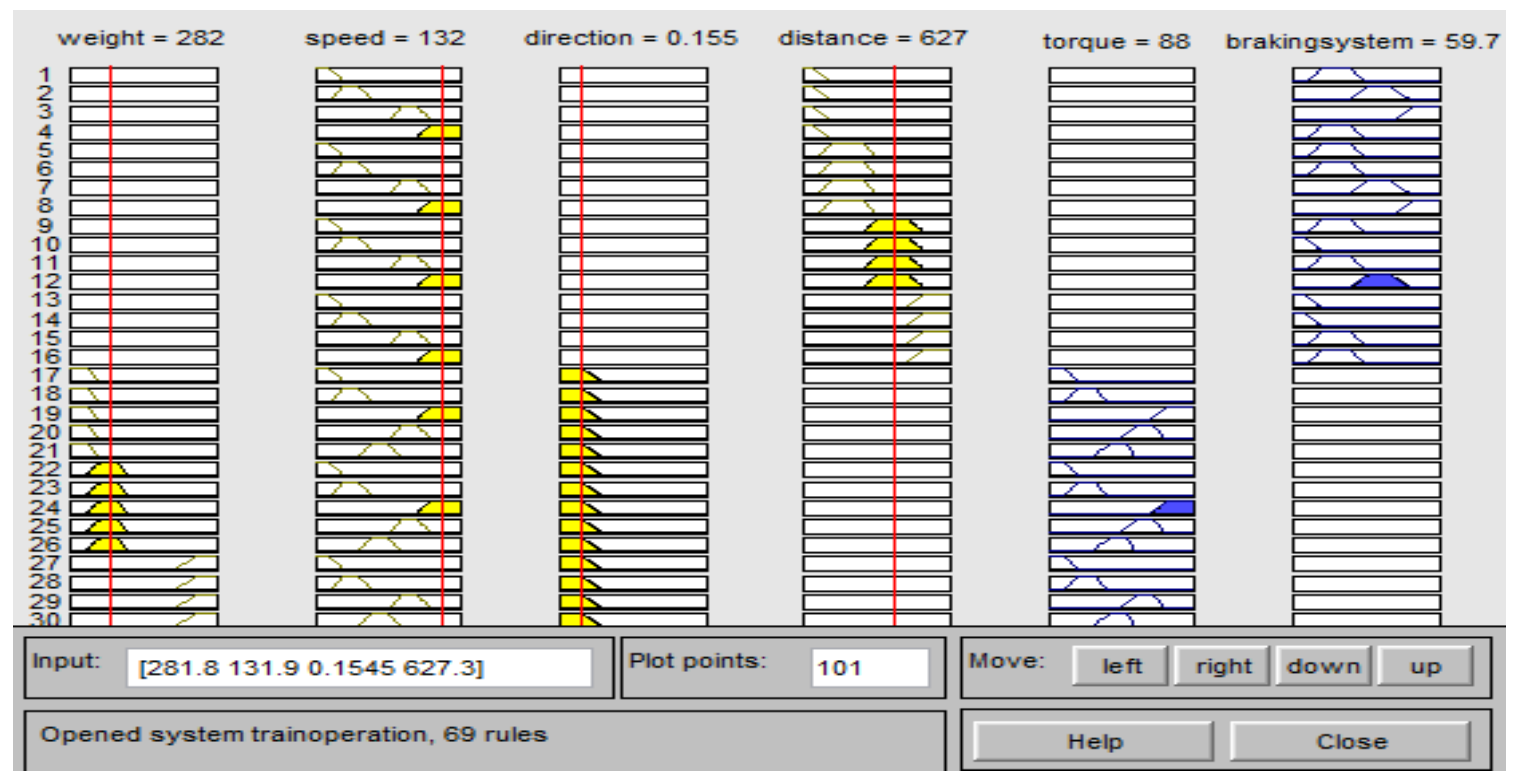

Figure 9: Rule Viewer Corresponding to Inputs and Outputs

Fig 9 shows rules regarding the inputs and outputs. In this case the weight is kept low, speed as very high, direction as forward and distance as far, so the resultant torque appears to be very high and braking system as heavy.

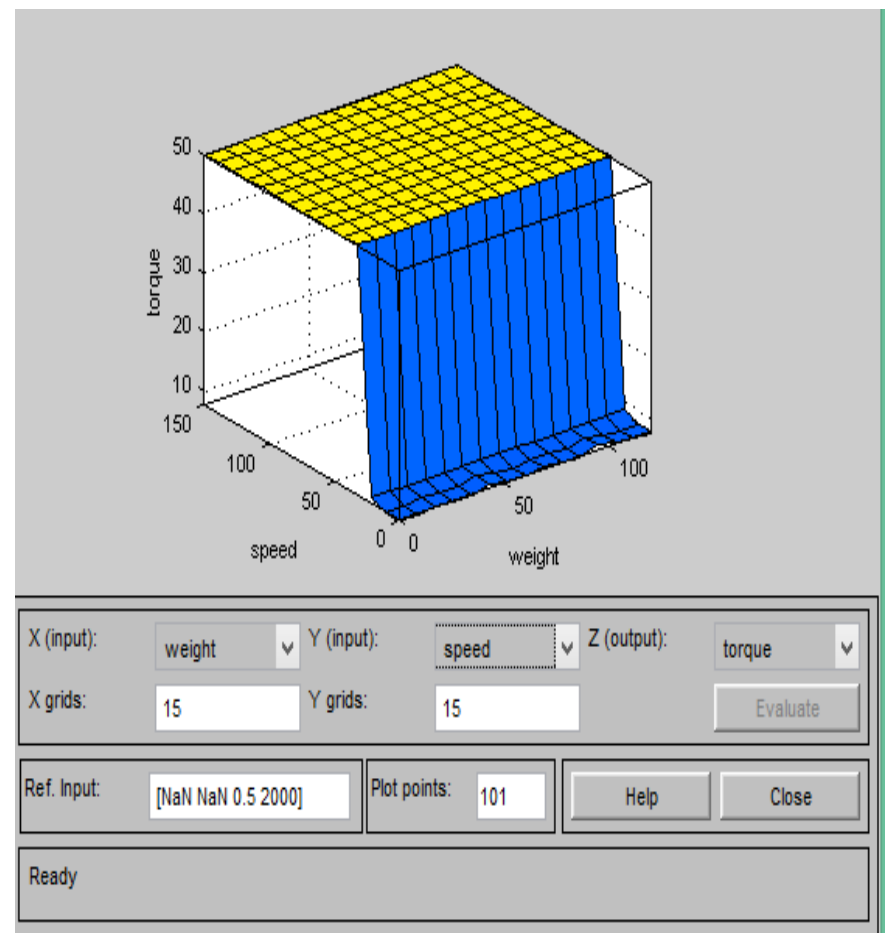

Figure 10: Surface Viewer

Fig 10 shows the surface view with inputs as speed and weight, and output torque.[8]

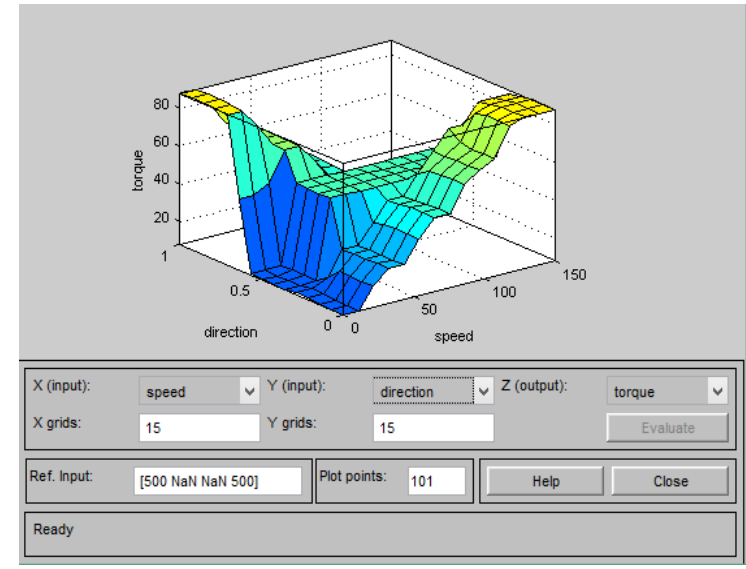

Figure 11: Surface Viewer

Fig 11 shows surface view with inputs as direction and speed, and output torque.[8]

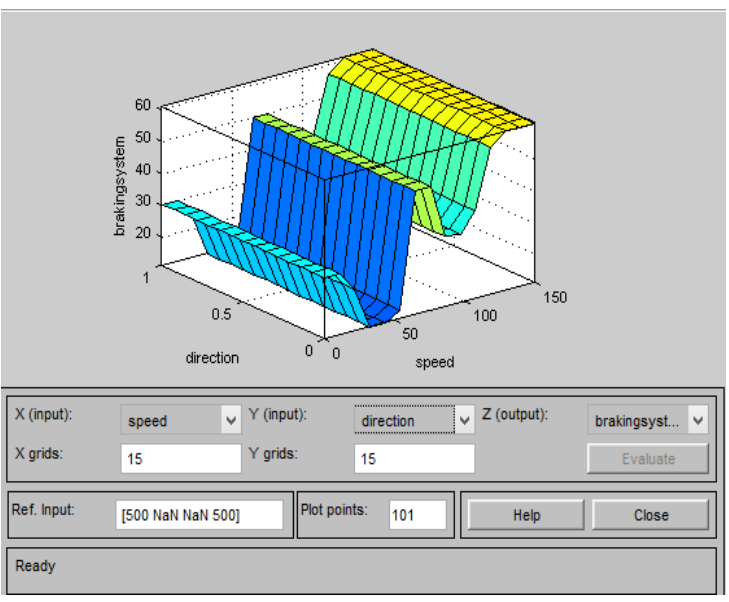

Figure 12: Surface Viewer 
Fig 12 shows surface view with inputs as direction and speed, and output braking system.[8]



Figure 13: Surface Viewer

Fig 13 shows surface view with inputs as weight and direction, and output torque.[8]

The results shows how output variables are dependent on input variables. [8]

Table 1: Variation in Torque w.r.t. Weight, Speed and Direction

\begin{tabular}{|l|l|l|l|}
\hline $\begin{array}{l}\text { WEIGHT } \\
\text { tonnes }\end{array}$ & $\begin{array}{l}\text { SPEED } \\
\text { In Kmph }\end{array}$ & DIRECTION & TORQUE \\
\hline 0 & 0 & 0 & 7.54 \\
\hline 20 & 15.2 & 0.0933 & 7.8 \\
\hline 40 & 31 & 0.0933 & 25.8 \\
\hline 50 & 44.5 & 0.19 & 25.8 \\
\hline 70 & 61.5 & 0.19 & 46.4 \\
\hline 90 & 75 & 0.153 & 46.7 \\
\hline 110 & 93 & 0.153 & 65.6 \\
\hline 120 & 105 & 0.153 & 67.5 \\
\hline
\end{tabular}

Table 1 shows variations in torque with respect to weight, speed and direction. Here direction is taken as forward so speed will be proportional to torque. It can be seen that, as speed increases torque also increases.

Table 2: variation in Torque w.r.t. Weight, Speed and Direction

\begin{tabular}{|c|c|c|c|}
\hline $\begin{array}{c}\text { WEIGHT } \\
\text { tonnes }\end{array}$ & $\begin{array}{c}\text { SPEED } \\
\text { In Kmph }\end{array}$ & DIRECTION & TORQUE \\
\hline 0 & 0 & 0.817 & 88.2 \\
\hline 19.6 & 15 & 0.817 & 87.9 \\
\hline 31.5 & 30.6 & 0.817 & 65.8 \\
\hline 52.3 & 45 & 0.82 & 65 \\
\hline 75.6 & 60.6 & 0.82 & 46.5 \\
\hline 90.2 & 75 & 0.849 & 46.2 \\
\hline 100.5 & 89.4 & 0.849 & 25.6 \\
\hline 119.8 & 105 & 0.849 & 25.4 \\
\hline
\end{tabular}

Table 2 shows variations in torque with respect to weight, speed and direction. Here direction is taken as backward so speed will be inversely proportional to torque. It can be seen that, as speed increases torque decreases.

Table 3: Variation in Braking System w.r.t. Distance and Speed

\begin{tabular}{|c|c|c|}
\hline $\begin{array}{c}\text { DISTANCE } \\
\text { In } \mathrm{Km}\end{array}$ & $\begin{array}{c}\text { SPEED } \\
\text { In Kmph }\end{array}$ & BRAKING SYSTEM(\%) \\
\hline 0 & 0 & 30.3 \\
\hline 1070 & 16.1 & 30.6 \\
\hline 2024 & 30.6 & 30.3 \\
\hline 3010 & 45 & 30.3 \\
\hline 3056 & 62.5 & 50 \\
\hline 3956 & 150 & 75.2 \\
\hline
\end{tabular}

Table 3 shows variation in braking system with respect to distance and speed. Braking system depends on both distance and speed.

\section{CONCLUSION}

In this paper an attempt has been made to design Mamdani type fuzzy rule-based illumination controller. Automatic train system is being implemented using fuzzy logic and the desired output has been observed. The use of fuzzy logic controller gives a smooth braking system. Developing and testing a prototype model of fuzzy logic controller can further prove the accuracy of system for breaking in trains. For a future development of this project, the Fuzzy Logic controller designed can be enhanced by applying mere rules. By then, it can produce better response. The response should be better and can be applied to a real hardware model to observe the real response and yet can improve the system.

\section{ACKNOWLEDGEMENT}

The authors thank the SDME Society, The Principal, Deans, The HOD(ECE), Faculty \& Non-Teaching Staff of Department, Family \& Friends for encouraging us for this research work. We especially thanks Prof. Siddalingesh S. Navalgund who taught us the course on Fuzzy Logic Control. Thanks are also due to the unknown referees whose critical reviews help improve the quality of the paper.

\section{REFERENCES}

[1] E. Khmelnitsky, “On an optimal control problem of train operation”, IEEE Transactions on Automatic Control, Vol.45, No.7, Pp.12571266, 2000.

[2] G. Chen and T.T. Pham, "Introduction to fuzzy systems", CRC Press, 2005.

[3] T. J. ROSS, “Fuzzy Logic With Engineering Applications”, 1997.

[4] P.S. Raju, F.J. Nikhath and S. Farook, “Automatic Train Operation And Control Using Matlab”, Vol.2, No.1, 2013.

[5] G. Sankar and S.S. Kumar, "Fuzzy logic based automatic braking system in trains”, International Conference on Power Electronics, Pp.383-387, 2006.

[6] ML Sharma and Sheetal Atri, "Fuzzy Rule based Automatic Braking System in Train using VHDL", International Journal of Computer Science and Technology, Vol. 2, Issue 2, Pp. 332-335, 2011.

[7] A. Siahvashi and B. Moaveni, "Automatic train control based on the multi-agent control of cooperative systems”, The Journal of Mathematics and Computer Science, Vol.1, No.4, Pp.247-257, 2010.

[8] The documentation on "FuzzyLogic Toolbox and surface view" http://www.mathworks.com. 


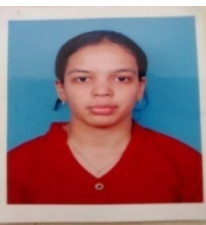

Meghna Madhava is pursuing her 4th year of undergraduate studies in the Dept. of E\&CE, at SDMCET, Dharwad. Her research interests include VLSI and Fuzzy Logic Applications.

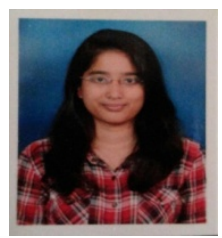

N. Meghana is pursuing her 4th year of undergraduate studies in the Dept. of E\&CE, at SDMCET, Dharwad. Her research interests include VLSI and Fuzzy Logic Applications

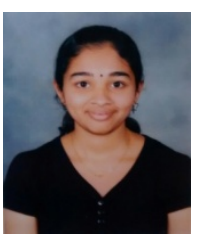

Mulpuru Supriya is pursuing her 4th year of undergraduate studies in the Dept. of E\&CE, at SDMCET, Dharwad. Her research interests include ITC and Fuzzy Logic Applications.

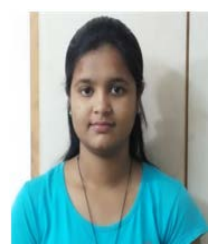

Divya is pursuing her 4th year of undergraduate studies in the Dept. of E\&CE, at SDMCET, Dharwad. Her research interests include VLSI and Fuzzy logic application.

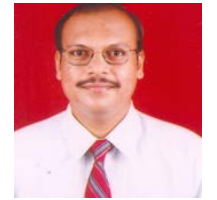

Siddalingesh S. Navalgund received Bachelor of Engineering in Electronics and Communication Engineering from Karnataka University Dharwad in 2000 and Master of Technology in Microelectronics and Control Systems from Visvesvaraya Technological University, Belgaum in 2004.

After spending two years in the industry, he joined as a faculty in N. M. A. M. in Institute of Technology, Nitte, Karnataka. Since 2005, he is serving as faculty in the Dept. of E\&CE, S. D. M. College of Engineering and Technology, Dharwad, Karnataka. His main research interests include VLSI design, embedded \& fuzzy systems and digital signal processing.

Mr. Navalgund is currently the life member of Institution of Electronics and Telecommunication Engineers (IETE), Indian Society for Technical education (ISTE) and Institution of Engineers India (IE-I). 